\title{
Adli, Mazda (2017): Stress and the City. Warum Städte uns krank machen. Und warum sie trotzdem gut für uns sind
}

\author{
München: C. Bertelsmann Verlag. 384 Seiten, 30 s/w Abbildungen
}

\section{Stefan Fina ${ }^{1}(\mathbb{D}$}

Online publiziert: 18. Januar 2018

๑) Springer-Verlag GmbH Deutschland, ein Teil von Springer Nature 2018

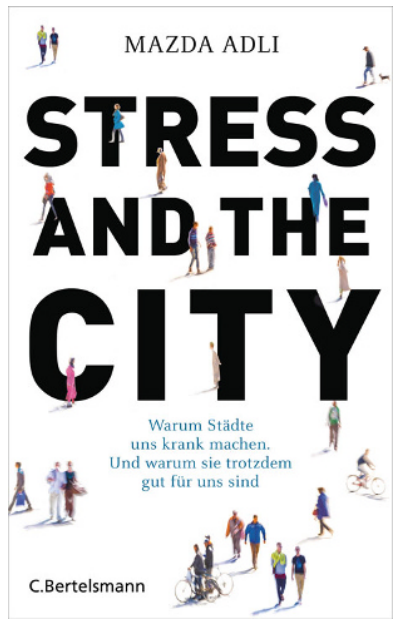

Im Dezember 2017 wurden Forschungsergebnisse aus der bislang größten Studie zu den regionalen Unterschieden im Auftreten und der Häufigkeit psychischer Erkrankungen, der sogenannten EU-GEI Studie, veröffentlicht. Die Ergebnisse und ihre Interpretation, veröffentlicht in der Zeitschrift „Jama Psychiatry“, bestätigen eine Reihe von Erkenntnissen, die mit dem medienwirksamen Etikett des ,Stadtneurotikers“ spätestens seit Woody Allens weltberühmtem Filmerfolg aus den 1970er-Jahren weitläufig bekannt geworden sind: Stadtbewohner, und darunter insbesondere die Bewohner von Großstädten, leiden statistisch gesehen sehr viel häufiger an psychischen Krankheiten als Landbewohner (Paris: 46 von 100.000 Einwohnern; ländlicher Raum in Spanien: sechs von 100.000 Einwohnern; Jongsma/GayerAnderson/Lasalvia et al. 2017).

Prof. Dr. Stefan Fina

stefan.fina@ils-forschung.de

1 ILS - Institut für Landes- und Stadtentwicklungsforschung, Brüderweg 22-24, 44135 Dortmund, Deutschland
Die Frage, die sich seither stellt, und die letztlich auch die oben genannte Studie nicht beantworten kann, lautet: Liegt dies daran, dass Stadtbewohner besonderen Belastungen ausgesetzt und damit anfälliger sind? Oder gehen Stadtbewohner einfach häufiger zum Psychiater? Vermutet wird, dass eine Kombination dieser beiden Erklärungsansätze zutrifft. Und, dass eine ganze Reihe von moderierenden Faktoren zu berücksichtigen ist. Dazu gehört die genetische Anfälligkeit für spezifische psychische Krankheiten, schichtspezifische Lebensweisen (Ernährung, Bewegung, Konsumverhalten) oder die Ausprägung von Umweltbelastungen über die gesamte Wohnbiographie eines Erkrankten. Die beiden letzteren Erklärungsansätze (soziale Lage, Umweltbelastungen) werden seit jeher als Forschungsthemen der Raumwissenschaften untersucht. Maßgebende Arbeiten finden sich in Standardwerken der Stadtsoziologie. Die Raumbedeutsamkeit von Umweltbelastungen wurde spätestens durch die Arbeiten von Ebenezer Howard als Reaktion auf den Londoner Smog der Jahrhundertwende vom 19. zum 20. Jahrhundert zum Gegenstand der Stadtforschung und hat letztlich zu unserer heutigen Umweltberichterstattung geführt. Interessanterweise wird aber auch die genetische Anfälligkeit für psychische Krankheiten mit raumstrukturellen Eigenschaften in Verbindung gebracht. Epigenetikern zufolge manifestieren sich bestimmte extreme Formen der Exposition städtischer Bevölkerungsgruppen gegenüber Stadtstress in den Gehirnstrukturen der Betroffenen und werden als Prädisposition für psychische Erkrankungen sogar weitervererbt (vgl. z. B. Allis/ Jenuwein/Reinberg 2007).

Diesem Thema und seinen zugrunde liegenden Erklärungsansätzen widmet sich das Buch „Stress and the City“ des Berliner Psychiaters und Psychotherapeuten Mazda Adli, das im Frühjahr 2017 im C. Bertelsmann Verlag erschienen ist. Adli ist Chefarzt der Fliedner-Klinik Berlin und Leiter des Forschungsbereichs „Affektive Störungen“ 
der Berliner Charité. Das Thema ist sein Steckenpferd. Mit großer Faszination vor den kulturellen Errungenschaften des Stadtlebens beleuchtet er die psychologischen Grundlagen des Stresserlebens in Großstädten. Einführend werden die landläufigen negativen Konnotationen mit dem Begriff „Stress“ relativiert: Stress kann durchaus positiv wirken, teilweise suchen und brauchen wir ihn regelrecht als Stimulanz und Motivation für alltägliches Handeln und kreativen Ansporn. Erst ein andauerndes Übermaß von Reizen, die das Gehirn nicht mehr verarbeiten kann, erzeugt psychische Belastungsstörungen. Die ersten einführenden Kapitel beschreiben diese Wirkmechanismen, unterfüttert mit den Erkenntnissen von Evolutionspsychologie und verwandter Disziplinen, sehr anschaulich.

Damit ist die Kernfrage dieses Buches beschrieben, die für die Raumwissenschaften wichtige Anschlussfragen aufwirft: Die Anziehungskraft von Großstädten wirkt ungebrochen auf bestimmte Bevölkerungsgruppen, die diese Reize und die mit der Dichte einer Stadt einhergehenden kulturellen und infrastrukturellen Angebote suchen - auch auf die Gefahr hin, dass das Stadtleben gesundheitliche Risiken birgt. Impulse für die Raumforschung ergeben sich dahingehend, dass nach Stand der Forschung aktuell zwei Gruppen die Reurbanisierung deutscher Großstädte antreiben: internationale Migranten und junge Menschen im Ausbildungsalter (vgl. Busch 2016). Die Wanderungssalden anderer Gruppen sind tatsächlich seit einigen Jahren mehrheitlich negativ, und es stellt sich im Hinblick auf Gentrifizierung und neue Tendenzen der Suburbanisierung die Frage, ab welchem Belastungspunkt die Standortwahl als ein Ausweichen vor den aktuellen Entwicklungen in den Kernstädten zu begreifen ist. Thesen dazu gibt es reichlich: Möglicherweise liegt der Wegzug an kulturell unterschiedlich gelagerten Stresstoleranzen für soziale Dichte oder an den geringeren Anpassungskapazitäten für Stadtstress bei Familien mit Kindern und älteren Menschen. Nicht zu vergessen die Preisspirale, die auf den Wohnungsmärkten vieler attraktiver Innenstadtlagen eine Befriedigung von Ansprüchen an Wohnraum und Lebensqualität für weniger zahlungskräftige Nachfrager verhindert. Auch wenn das Buch sich nicht unmittelbar auf diese Fragen konzentriert, liefern die nächsten Kapitel aus der Sicht der Psychologie aufschlussreiche Erklärungsansätze zum Beispiel zum Territorialverhalten von Stadtbewohnern, zur Gruppendynamik in sozialen Schichten, zur Wahrnehmung von Gefahren und der Rolle von Verkehrssystemen, zu planerischen Interventionen einer gesundheitsfördernden Stadtentwicklung oder zu den Auswirkungen von sozialer Dichte auf das Wohlbefinden von Stadtbewohnern. Letztlich sind diese Ausführungen für alle interessierten Stadtforscher eine erfrischende Perspektive und bergen das Potenzial, raumwissenschaftliche Thesenbildungen künftig mit Ansätzen aus der Gesundheitsforschung zu bereichern. Mit dem Netzwerk Neu- rourbanistik, das von Adli maßgeblich mit initiiert wurde, besteht hierfür eine begrüßenswerte Vernetzungsplattform für den interdisziplinären Austausch von Stadtforschern und Gesundheitswissenschaftlern, die diesen Fragen zukünftig sehr viel intensiver nachgehen wollen.

Neben aller wissenschaftlichen Recherchearbeit präsentiert dieses Buch auch sehr persönliche Erfahrungen und verlässt damit die Ebene der reinen Fachliteratur. Auf den knapp 400, mit gelungenen Grafiken bebilderten Seiten, unterfüttert Adli die klug ausgewählten Forschungsergebnisse zum Thema mit Protokollen von Interviews, die mit Experten geführt wurden. Neben prominenten Architekten und Wissenschaftlern aus der Stadtsoziologie (unter anderem Richard Sennett) sind darunter auch Persönlichkeiten des öffentlichen Lebens, zum Beispiel der Opern- und Theaterregisseur Barry Kosky oder die ehemaligen Bürgermeister von Bogota und Barcelona. Die eigenen Erfahrungen des Autors (aufgewachsen in Teheran, Bonn und Köln, Aufenthalte in Wien und New York, beruflich beheimatet in Berlin) erklären nicht nur sein genuines Forschungsinteresse, sondern liefern vielfach auch Einblicke in die Motivation und die induktive Herangehensweise an den Forschungsgegenstand in der Psychologie. Für ausgewählte Beobachtungen werden erklärende Regeln gesucht und im Experiment überprüft. Die Ergebnisse werden dann im Hinblick auf die Eingangsfragen interpretiert. Hier setzt ein kleiner Punkt der Kritik an. Teilweise wirken diese Interpretationen ein wenig spekulativ beziehungsweise werden zu vorschnell verallgemeinert. Ein Beispiel ist ein Studienergebnis aus Boston, das scheinbar den Zusammenhang zwischen dem sozialen Zusammenhalt einer Gesellschaft und der Dichte von Baumkronen im Wohnumfeld nachweist. Im kontrollierten Setting der Studie mag diese Korrelation zwar signifikant gewesen sein, dadurch gilt dieser Zusammenhang aber noch nicht überall. Hier sollte die interdisziplinäre Zusammenarbeit zwischen Gesundheits- und Raumwissenschaften zukünftig ansetzen. Aus der Sicht des Rezensenten bieten neue Technologien zur Erhebung nutzergenerierter Daten das Potenzial, einen methodischen Quantensprung zu schaffen. Bei aller Vorsicht bei der Qualitätssicherung von Daten aus sozialen Medien und anderen Informationsplattformen können heute Experimente und Studiendesigns einer sehr viel größeren Anzahl an Probanden vorgesetzt und häufiger wiederholt werden. Damit kann eine Abdeckung auch im Sinne unterschiedlicher räumlicher Settings erreicht werden. Mazda Adlis „Stress and the City“ bietet neben all seinen Qualitäten einer kurzweiligen und trotzdem hochinformativen Lektüre zahlreiche Impulse, um neue Ideen für dieses spannende Forschungsfeld zu generieren.

Und übrigens: Erstaunlicherweise zeigt die eingangs erwähnte Studie aus England, dass ein bislang kaum berücksichtigter Faktor eine signifikante Rolle für die Häufigkeit psychischer Krankheiten zu spielen scheint: Menschen, die 
im Wohneigentum leben, zeigen deutlich geringere Häufigkeiten psychischer Krankheitsbilder als Menschen, die zur Miete leben. Also sollte künftig die Bildung von Wohneigentum als wichtige gesundheitsfördernde Maßnahme in der Stadtpolitik etabliert werden? Kann sein, dass dies helfen würde - muss aber nicht.

\section{Literatur}

Allis, C. D.; Jenuwein, T.; Reinberg, D. (Hrsg.) (2007): Epigenetics. Cold Spring Harbor, NY.
Busch, R. (2016): Inländische Wanderungen in Deutschland - Wer gewinnt und wer verliert? In: Zeitschrift für Immobilienökonomie 2, 2, 81-101. doi: 10.1365/s41056-016-0012-3

Jongsma, H. E.; Gayer-Anderson, C.; Lasalvia, A.; Quattrone, D.; Mulè, A.; Szöke, A.; Selten, J.-P.; Turner, C.; Arango, C.; Tarricone, I.; Berardi, D.; Tortelli, A.; Llorca, P.-M.; de Haan, L.; Bobes, J.; Bernardo, M.; Sanjuán, J.; Santos, J. L.; Arrojo, M.; Del-Ben, C.-M.; Rossi Menezes, P.; Murray, R. M.; Rutten, B.P.; Jones, P. B.; van Os, J.; Morgan, C.; Kirkbride, J. B. (2017): Treated incidence of psychotic disorders in the multinational EU-GEI study. In: JAMA Psychiatry. doi: 10.1001/jamapsychiatry.2017.3554 (online first). 